\title{
Características de la germinación de Lippia palmeri (Wats) proveniente de regiones silvestres del desierto de Altar, Sonora, México
}

\author{
Germination characteristics of Lippia palmeri (Wats) \\ from wild desert regions of Altar, Sonora, México \\ Jesús Borboa-Flores ${ }^{1}$, María Magdalena Ortega-Nieblas ${ }^{2}$, Diana McCaughey-Espinoza², \\ María del Refugio Robles-Burgueño ${ }^{3}$, Mercedes Serna-Félix ${ }^{1}$, Francisco Javier Cinco-Moroyoquie ${ }^{1}$, \\ Francisco Javier Wong-Corral ${ }^{1}$,Edgar Omar Rueda Puente ${ }^{*}$
}

\section{RESUMEN}

Existen varias especies del género de Lippia nativas e introducidas, que son de gran potencial en zonas áridas del noroeste de México; estas especies presentan bajo porcentaje de germinación debido a la dureza y/o dormancia de la semilla. El objetivo del presente estudio consistió en dilucidar el efecto del ácido giberélico sobre la germinación de la semillas silvestre de Lippia palmeri. Para ello se recolectó la semilla en dos regiones nativas (Puerto del Orégano y Santa Ana, Sonora). Se probaron tres concentraciones con ácido giberélico $\left(\mathrm{GA}_{3}\right)(100,200$ y 300 ppm, sometidas a tres tiempos de remojo de la semilla (30, 60 y 120 min.) y como control agua destilada. Para cada tratamiento se utilizaron 100 semillas por caja Petri por triplicado siendo un total de 72 cajas Petri colocadas a una temperatura ambiente de $27^{\circ} \mathrm{C}$ y $80 \%$ de humedad relativa. Para la concentración de $100 \mathrm{ppm}$ en los tres tratamientos de remojo la semilla de Puerto del Orégano, mostró un alto porcentaje de germinación (90, 100 y 100\%), para los tratamientos de 200 y 300 ppm el porcentaje de semilla germinada fue menor (80\%). La semilla de Santa Ana mostró el porcentaje más alto de germinación con (80\%) obtenido a 300 ppm y 120 minutos en remojo, el resto de los tratamientos no superó el 70\% de germinación. Se concluye que Lippia palmeri requieren ser estimuladas por la giberelina a fin de promover la acción enzimática que induce la ruptura de las sustancias de reserva de la semilla.

Palabras clave: germinación, Lippia palmeri, ácido giberélico $\left(\mathrm{GA}_{3}\right)$.

\begin{abstract}
There are several species of native and introduced Lippia, which are of great potential in arid areas of Northwest Mexico; these species have low germination percentage due to the hardness and / or seed dormancy. The aim of this study was to elucidate the effect of gibberellic acid on germination of seeds of wild Lippia palmeri. To do this, the seed was collected in two native sites (Puerto del Orégano and Santa Ana, Sonora). Three concentrations with gibberellic acid $\left(G A_{3}\right)(100,200$ and 300 ppm), subjected to three times of soaking the seed (30, 60 and 120 min.) were tested and as a control distilled water. For each treatment 100 seeds were used by Petri dish in triplicate with a total of 72 Petri plates placed in an ambient of $27^{\circ} \mathrm{C}$ and $80 \%$ relative humidity. For the concentration of 100 ppm in the three treatments soaking seed port oregano, showed a high percentage of germination (90, 100 and 100\%); for treatments of 200 and 300 ppm the percentage of germinated seed was lower (80\%). The seed of Santa Ana showed the highest percentage of germination (80\%) obtained 300 ppm and 120 minute soak; the other treatments did not exceed $70 \%$ germination. It is concluded that Lippia palmeri require stimulated by giberalina to promote the enzymatic action inducing breakdown of reserve substances seed.
\end{abstract}

Key words: germination, Lippia palmeri, Giberelic acid $\left(G_{3}\right)$

\footnotetext{
1 Departamento de Investigación y Postgrado en Alimentos. Universidad de Sonora. Hermosillo, Sonora, México.

2 Departamento de Investigaciones Científicas y Tecnológicas de la Universidad de Sonora. Hermosillo, Sonora, México.

3 Centro de Investigación en Alimentación y Desarrollo A.C. CIAD- Hermosillo, Sonora, México.

4 Departamento de Agricultura y Ganadería. Universidad de Sonora. Hermosillo, Sonora, México.

* Autor de correspondencia: erueda04@santana.uson.mx
}

Fecha de Recepción: 22 Marzo, 2016.

Fecha de Aceptación: 4 Agosto, 2016.

DOI: $10.4067 / \mathrm{S} 0718-34292016005000021$ 


\section{Introducción}

En los desiertos y las zonas áridas del noroeste de México, se han originado y evolucionado una gran cantidad de especies, lo que contribuye a la diversidad biológica y genética (Allison et al., 2015). Por las condiciones ambientales prevalentes en estas zonas, las especies vegetales que ahí se desarrollan representan un recurso genético de tolerancia a factores ambientales extremos como lo son la sequía, salinidad, bajas y altas temperaturas, alta radiación, entre otros (Bashan et al., 2000). No obstante lo anterior, en algunas comunidades rurales aledañas a los desiertos, como en el de Altar, Sonora, la vegetación de ese desierto es el soporte de los sistemas de producción pecuarios y agroforestales, pues se aprovecha la producción de biomasa y la posibilidad de obtención de productos procesados en donde se distingue la especie de orégano Lippia palmeri. Estos ecosistemas desérticos tienen una alta fragilidad y si se considera cada vez mayor variabilidad en el comportamiento del clima a nivel mundial existe un alto potencial de pérdida de los recursos vegetales biótico y genético que se desarrollan en esas áreas (Rueda et al., 2004). Diversas instituciones nacionales e internacionales llevan a cabo investigación sobre flora de desiertos donde también se incluyen a las zonas áridas y es necesario que los resultados de trabajos de investigación y aprovechamiento de la flora, se difundan de manera directa entre investigadores y productores para que el conocimiento generado sea empleado en el aprovechamiento sustentable de las especies vegetales de las zonas áridas.

Por tales motivos se describe que en el desierto de Altar, Sonora, en el noroeste de México, el Orégano (Lippia palmeri Wats), es una de las especies forestales potenciales para ser explotado comercialmente (González et al., 2007); todas las poblaciones que actualmente existen son silvestres correspondiendo a una misma especie, que es la de palmeri, la que se caracteriza por ser de porte arbustivo midiendo de 0,5 a 2,5 metros de alto, con tallos leñosos cerrados, sus hojas son pecioladas, ovadas de 1 a $2,5 \mathrm{~cm}$ de longitud y de la cual se observa una gran diversidad en las dos regiones silvestres seleccionados en su recolecta (Santa Ana, Sonora y Puerto del Orégano, México).

La búsqueda de encontrar el método más apropiado para incrementar la germinación de diferentes especies de semillas silvestres ha sido una tarea difícil cuando se pretende la introducción de un cultivo de una especie con fines comerciales (Lambert et al., 2001; Blanco et al., 2005). Sin embargo, la explotación de este recurso lo hace susceptible de ser eliminado de su hábitat natural, si su aprovechamiento no es adecuado.

La investigación y desarrollo de producción de plantas silvestres requieren de un manejo estratégico el que consiste en lograr una colecta en época de producción de semilla y análisis de las semillas a nivel laboratorio para determinar su calidad (ISTA, 1999). Estos análisis se componen de pruebas de germinación que precisa la viabilidad de la semilla, la cual considera la madurez en el momento de la recolección (Nair, 1997).

Algunas especies, presentan bajos porcentajes de germinación debido a la dureza de su testa, pero varios estudios han mostrado que la aplicación de ácido giberélico $\left(\mathrm{AG}_{3}\right)$ aumenta la capacidad de germinación de las semillas y frecuentemente reemplaza la necesidad de estímulos ambientales, como la luz y la temperatura (Ungar, 2000). El ácido giberélico, es una sustancia que confiere a la semilla el desarrollo del talluelo de que emerge la semilla, a su vez incentiva la síntesis de proteínas en el proceso de germinación de las semillas, así como las auxinas incentivan el desarrollo de la radícula (Hartmann et al., 1997).

El presente estudio consistió en analizar los efectos del ácido giberélico sobre la germinación de semilla silvestre de orégano (Lippia palmeri Wats) de dos procedencias, a tres tiempos de remojo y tres concentraciones a temperatura y humedad de ambiente.

\section{Materiales y Métodos}

Se estudió la multiplicación sexual del orégano (Lippia palmeri Wats) procedente de dos regiones (Puerto del Orégano y Santa Ana, Sonora, México). La región de Puerto del Orégano, es localizado a $29^{\circ} 02^{\prime} 52,1^{\prime \prime}$ Norte y a $110^{\circ} 40^{\prime} 15,8^{\prime \prime}$ Oeste a $31 \mathrm{~km}$ carretera Hermosillo a Mazatán. Por su parte el área de Santa Ana, Sonora está situado a un altitud de $380 \mathrm{msnm}$, y a una latitud de $\left(27^{\circ}\right.$ $01^{\prime} \mathrm{N}$ ) y longitud (108 $\left.56^{\prime} \mathrm{O}\right)$ (INEGI, 2016). Las semillas fueron recolectadas en junio del 2015. Se probaron tres tiempos de remojo de la semilla con ácido giberélico $\left(\mathrm{GA}_{3}\right)$ por $(30,60$ y $120 \mathrm{~min}$.) a tres concertaciones $(100,200$ y 300 ppm) y como control agua destilada. 
Para conocer la influencia del ácido giberélico sobre la germinación de las semillas, se utilizaron 100 semillas secas previamente identificadas y cuidando que no tuvieran daños mecánicos ni de insecto, estas se colocaron en (cajas Petri) y se cubrieron con ácido giberélico en las proporciones antes mencionadas. Durante el período de tratamiento las semillas fueron agitadas regularmente con el fin de obtener resultados uniformes. Al final del período de cada remojo o tratamiento, se escurrió el ácido giberélico; las semillas fueron sembradas, aplicando 100 semillas por placa Petri con tres réplicas y un control de 100 semillas remojadas en agua, las semillas fueron ubicadas al interior de la cajas sobre y bajo papel húmedo utilizando como sustrato papel filtro, algodón y papel estraza. Las placas fueron colocadas a temperatura ambiente del laboratorio la cual oscilaba de $25 \pm 2{ }^{\circ} \mathrm{C}$ y de $80 \%$ de humedad relativa, utilizando un medidor Thermo-Hygro DTH 880, por un período de 20 días; al tercer día se observaron las primeras semillas germinadas, realizándose conteos de plántulas germinadas cada dos días (ISTA, 1999). En esta prueba se consideraron tres características: plántulas normales germinadas, valor germinativo de la semilla y semillas no germinadas en su caso (Gulzar y Khan, 2001).

El porcentaje de germinación fue calculado tomando en cuenta el número de semillas germinadas entre el número de semillas totales puestas a germinar; para la obtención del valor germinativo, se tomó en cuenta el valor más alto de germinación el que fue multiplicado por los días medios de la germinación, este valor nos representa la fase lenta y rápida de la germinación.

\section{Diseño experimental}

En el presente estudio se utilizó un diseño estadístico completamente al azar. Utilizando este mismo diseño se realizaron análisis de varianza del porcentaje de germinación transformando previamente los valores porcentuales con arcoseno (Sokal y Rohfl, 1988). La diferencia mínima significativa entre las medias de los tratamientos, de las variables estudiadas (porcentaje de germinación), fueron evaluadas mediante la prueba de Rango Múltiple de Duncan al 0,05\%. Los datos fueron analizados utilizando el programa estadístico de cómputo SAS 9.1 (SAS, 2015).

\section{Resultados y Discusión}

De acuerdo a la metodología anteriormente planteada, los resultados se muestran a continuación.

\section{Prueba de germinación con germoplasma de Santa Ana, Sonora}

Se puede observar en la Figura 1, se presentan los resultados obtenidos mediante diagramas de caja, los que muestran que la variación en los porcentajes de germinación para los diferentes tratamientos son similares, a excepción del tratamiento I (300 ppm durante 120 minutos de remojo), ya que se tuvo un $80 \%$ de germinación total, superando significativamente al resto de los tratamientos que presentaron el $70 \%$.

La semilla de la región de Santa Ana, Sonora contienen el $4.2 \%$ de humedad, presentó el $60 \%$ de germinación (Figuras 1 y 2 y Tabla 1 ), resultado obtenido de 100 ppm de ácido giberélico y en remojo por 60 y 120 minutos (B) y de (D y F) al utilizar 200 ppm de ácido giberélico en remojo. El valor más alto fue de $80 \%$ de germinación (I), resultado obtenido al usar 300 ppm del ácido giberélico en remojo por 120 minutos (Figura 1). Además, se obtuvo valores iguales de $70 \%$ de germinación en (A, C, E, G y H), estos valores que se obtuvieron en los tratamientos al usar 100 ppm, 200 ppm y 300 ppm del ácido giberélico y en los diferentes tiempos de remojos.

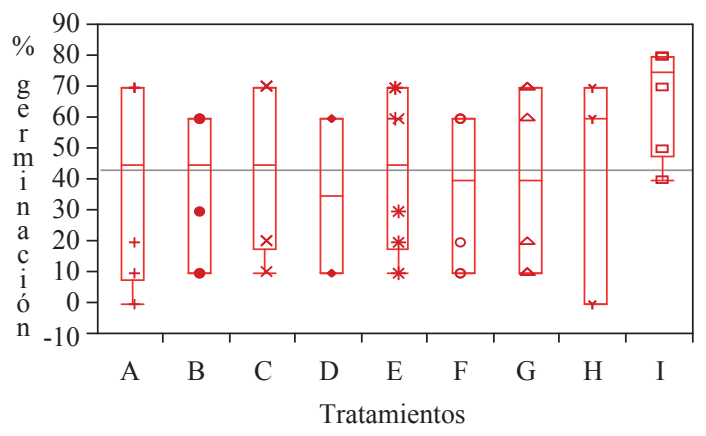

Figura 1. Resultados de los tratamientos con ácido giberélico de las semillas proveniente de la región de Santa Ana, Sonora. Tratamientos: Remojo en ácido giberélico, a una concentración de 100 ppm en tres tiempos: $\mathrm{A}=30 \mathrm{~min}$., $\mathrm{B}=60 \mathrm{~min}, \mathrm{C}=120$ min.; Remojo en ácido giberélico, a una concentración de 200 ppm en tres tiempos: $\mathrm{D}=30 \mathrm{~min}$., $\mathrm{E}=60 \mathrm{~min}$, $\mathrm{F}=120 \mathrm{~min}$; Remojo en ácido giberélico, a una concentración de 300 ppm en tres tiempos: $\mathrm{G}=30 \mathrm{~min}$., $\mathrm{H}=60 \mathrm{~min}$., $\mathrm{I}=120 \mathrm{~min}$. 


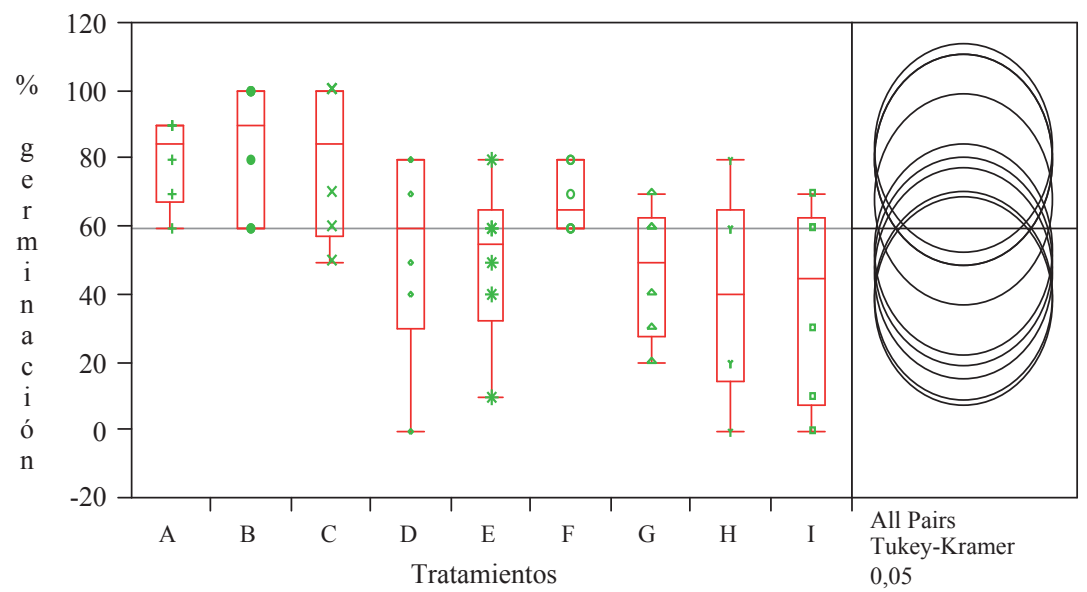

Figura 2. Resultados de los tratamientos con ácido giberélico de las semillas proveniente de la región de Puerto del Orégano, Sonora.

Tratamientos: Remojo en ácido giberélico, a una concentración de 100 ppm en tres tiempos: A = 30 min., $\mathrm{B}=60 \mathrm{~min}, \mathrm{C}=120 \mathrm{~min}$; Remojo en ácido giberélico, a una concentración de 200 ppm en tres tiempos: $\mathrm{D}=30 \mathrm{~min}$., $\mathrm{E}=60 \mathrm{~min} ., \mathrm{F}=120 \mathrm{~min}$.; Remojo en ácido giberélico, a una concentración de 300 ppm en tres tiempos: $\mathrm{G}=30 \mathrm{~min} ., \mathrm{H}=60 \mathrm{~min}$., $\mathrm{I}=120 \mathrm{~min}$.

Tabla 1. Resultados de germinación y de algunas características de la semillas de Lippia palmeri Wats (Orégano).

\begin{tabular}{lccccc}
\hline Región de muestreo & $\%$ germinación & $\%$ humedad & $\begin{array}{c}\text { Inicio de germinación } \\
\text { mínima-máxima }\end{array}$ & \# de semillas por kg & Fecha de colecta \\
\hline Puerto del Orégano & 70 & 2,52 & Tercer día & 666.000 & Junio 2015 \\
Santa Ana, Sonora & 60 & 4,2 & Sexto día & 3.000 .000 & Sep. 2015 \\
\hline
\end{tabular}

Testigo control $=0$ en los tres tiempos evaluados (30,60 y 120 min.) y en la diferentes concentraciones de ácido giberélico (100, 200 y 300 ppm).

\section{Región de Puerto del Orégano}

El porcentaje de germinación de las semillas de Puerto del Orégano, es diferente al observado en Santa Ana, como se observa en la Tabla 1. Los porcentajes al concluir la germinación variaron del 70 al 100\% (Figura 2), siendo los tratamientos de 100 ppm los que presentaron resultados más altos (A, B y C). Mientras que los tratamientos G e I, presentaron el $70 \%$ de germinación. El ANOVA indica que si existen diferencias significativas $(\mathrm{p}=0,0026)$ entre los tratamientos. La prueba a posteriori de Tukey Tabla 1, indica que los tratamientos se separan en dos grupos con características similares: el grupo de los tratamientos A, B, C y F que presentan una menor variación y mayor porcentaje de germinación que los del grupo D, E, G, H e I. El programa JMP, muestra estos resultados gráficamente (Figura 3) se presentan estos mismos resultados para su corroboración con respecto a la germinación obtenida en las dos regiones de muestreo.

Las semillas provenientes de Puerto Orégano contienen 2,5\% de humedad, semillas que presentaron mayor porcentaje de germinación, como se observa en los resultados de en la Tabla 1, donde los valores fueron altamente significativos presentando 90, 100 y $100 \%$ de germinación (Figura 2) resultado obtenido al usar la concentración de 100 ppm del ácido giberélico como se observa en los tratamientos (A, B y C); respecto al testigo, este reportó $0 \%$ de germinación no mostrado en la gráfica, situación que fue detectada en los restantes tiempos evaluados (30, 60 y 120 minutos de remojo) y en concentraciones de 100, 200 y 300 ppm de ácido giberélico (indicado al pie de figuras y tablas). Por otro lado los tratamientos (D, E y F) presentaron 


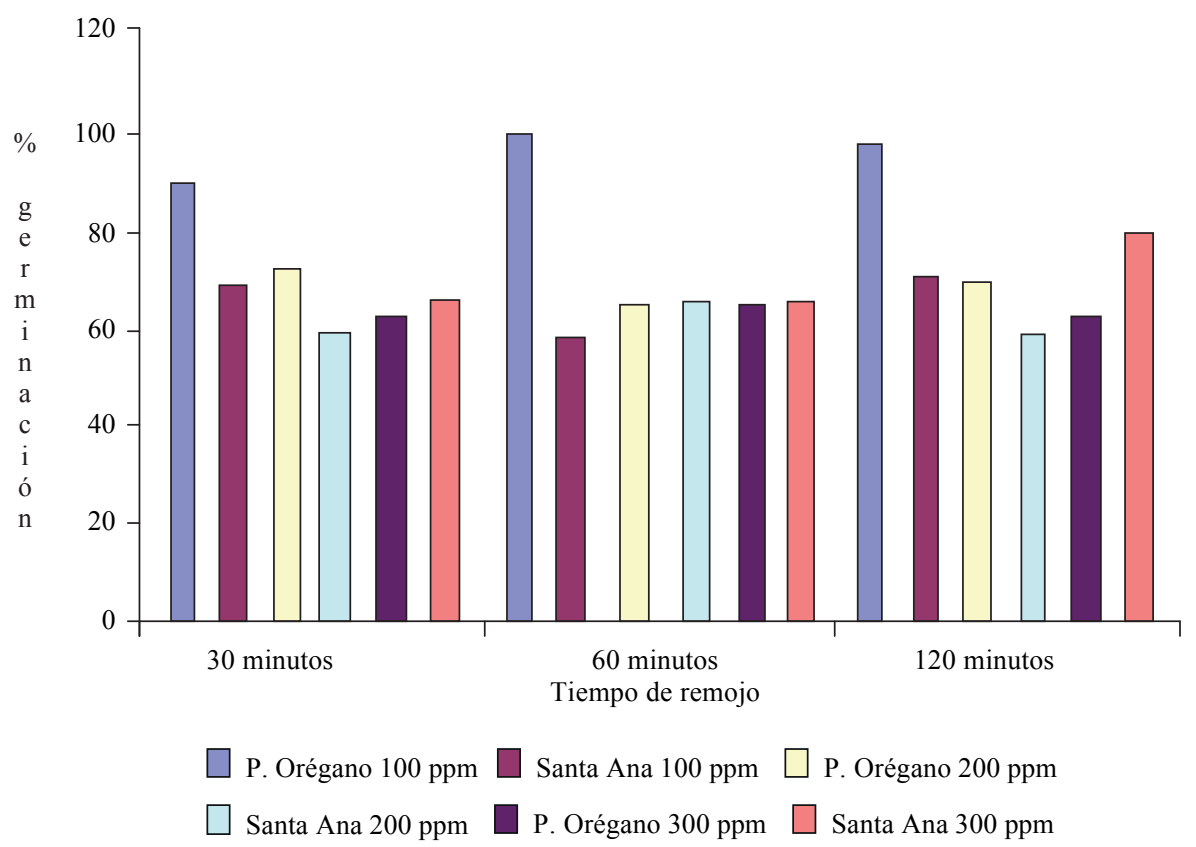

Figura 3. Comparación del porcentaje de germinación de Lippia palmeri por región de colecta.

igual porcentaje de germinación que fue de $80 \%$, al utilizar 200 ppm del ácido giberélico y utilizando los tres tiempos de remojo. Los valores registrados más bajos fueron los tratamientos a las semillas con 30, 120 y 60 min. de remojo en la concentración de 300 ppm de ácido giberélico alcanzando entre 60 y $80 \%$ de germinación, como se aprecia en la Figura 2 (G, H y I).

\section{Discusión}

En 2014 (Fuentes et al.), estudiaron la propagación de orégano (Ocimum gratissimum L.), utilizando diferentes tiempos de remojo con ácido giberélico en diferentes concentraciones 50, 100, $250,500,750$ y $1.000 \mathrm{ppm}$ y aplicaron un solo tiempo de remojo de 24 horas, los resultados que obtuvieron indican que el tiempo de remojo de 24 horas con agua corriente no resultaron efectivos, pero es posible mejorar la germinación con aplicaciones exógenas de ácido giberélico en concentraciones de $250 \mathrm{ppm}$. Asimismo, Hernández-Verdugo et al. (2005), detectaron que el ácido giberélico y ácido sulfúrico promueven la germinación en Chile silvestre remojándola y a distintas temperaturas. Resultados similares fueron identificados en el presente estudio, a llevar a cabo los tiempos de remojo en la semilla de orégano con las diferentes concentraciones del ácido giberélico; lo contrario fue observado en aquellos sin tratamiento (control) al no germinar. Datos no presentados.

Por su parte Nair (1997), considera que el porcentaje de germinación está influenciado por el tiempo de madurez de la semilla en las diferentes regiones de colecta, además de aquella vegetación con la que esta se asocia, pues una cantidad específica de exudados generados por los sistemas radiculares de las plantas en conjunto, promueve que microorganismos benéficos sean atraídos y jueguen un papel importante en la germinación de semillas. En este estudio la diferencia de porcentaje de germinación entre ambas regiones puede deberse, al tiempo que tarda la semilla en madurar en los dos lugares de estudio (Santa Ana y Puerto del Orégano), influyendo en este proceso las horas luz y la climatología del área.

\section{Conclusiones}

De acuerdo a los resultados obtenidos en el análisis estadístico empleado, y bajo las condiciones del laboratorio en que se llevó a cabo el presente estudio, se concluye que la aplicación del ácido giberélico fue exitoso por lograr la germinación en las semillas del orégano de Lippia palmeri, ya que el testigo reportó cero por ciento de germinación 
(remojadas en agua sin ácido giberélico) indicando, que las semillas requieren un tratamiento previo con la hormona en estudio, para lograr la inhibición de otras hormonas presentes y, por ende, la giberelina, podría promover la acción enzimático, que induce la ruptura de las sustancias de reserva de las semillas del orégano Lippia palmeri. Las semillas de la región de Puerto del Orégano fueron la más viable al proceso de germinación con la aplicación de ácido giberélico en su concentración de 100 ppm y en remojo por 30, 60 y 120 minutos, por lo que las semillas pueden ser utilizadas para trabajos de propagación masiva, mientras que las semillas del orégano provenientes de la región de Santa Ana,
Sonora, lograron su la germinación más alta con el uso de 300 ppm del ácido giberélico, en remojo por 120 minutos. La variación en germinación en cuanto a la región de recolecta de las semillas no garantiza que el vigor estas sea suficiente para su desarrollo y sobrevivencia por lo que se requiere realizar las pruebas. Es posible mejorar la germinación con aplicaciones exógenas de ácido giberélico a partir de concentraciones de 100 ppm. y usando los tres tratamientos de remojo $(30,60$ y 120 minutos). Estudios concernientes a etapas fenológicas posteriores deben ser considerados para generar un paquete tecnológico y transferirlos al sector productivo.

\section{Literatura Citada}

Allison, L.; Brown, J.; Haryward, H.; Richards, L. 2015. Suelos salinos y sódicos. Ed. Limusa. México, D.F. 215 p. Bashan, Y.; Moreno, M.; Troyo, E.

2000. Growth promotion of the seawater-irrigated oilseed halophyte Salicornia bigelovii inoculated with mangrove rhizosphere bacteria and halotolerant Azospirillum spp. Biology and Fertility of Soils, 32: 265-272.

Blanco, N.L.; Luengas Jiménez, B.C.; Bautista Barrón, B.E. 2005. Enraizamiento estacional de varetas de orégano (Lippia berlandieri Schawer). Revista Chapingo, serie Zonas Aridas, 4 (2): 25-30.

Fuentes, V.; Rodríguez, N.; Rodríguez, C.

2014. Acerca de la propagación de Ocimum gratissimum L. Rev. Cubana Plant Med, 1 (1): 3-7.

Hernández-Verdugo, S.; Sánchez- Peña, P.; Villarreal-Romero, M. 2005. Efecto del Ácido Giberélico, Ácido Sulfúrico, Remojo y la Temperatura: Variación en la Germinación entre Poblaciones de Chile Silvestre. Ciencia y Tecnología, 3 (2): 23-28.

Hartmann, H.T.; Kester, D.E.; Davies, F.T.L; Geneve, R. 1997. Hartmann \& Kester's Plant Propagation: Principles and practices. $6^{\text {Th }}$ Pretice Hall. 928 p.

ISTA (International Seed Testing Association). 1999. International Rules for Seed Testing, 1999. Seed Science and Technology, 21 Netherlands. $288 \mathrm{p}$.

INEGI Instituto Nacional de Estadística Geografica e Informatica). 2016. Atlas de México. Disponible en: URL: http://www. inegi.org.mx/ consultado el 25/Mar/2016.

Gulzar, S.; Khan, A.

2001. Seed Germination of Halophytic Grass Aeloropus logopoides. Annals of Botany, 87: 319-322.
González, G.M.C.; Soto, H.M.; Kite, G.; Martínez, V.M. 2007. Actividad antioxidantes de flavonoides del tallo de orégano mexicano (Lippia graveolens HBK var. berlandieri Schauer). Revista Fitotecnia Mexicana, 30 (1): 43-49.

Lambert, R.J.W.; Skandamis, P.N.; Coote, P.J.; Nychas, G.-J.E. 2001. A study of minimum inhibitory concentration and mode of action of oregano essential oil, thymol and carvacrol. Journal of Applied Microbiology. 91, 453-462.

Nair, P.K.R.

1997. Agroforestería. Centro de Agroforestería para el Desarrollo Sostenible y Universidad Autónoma Chapingo. Chapingo, México, D.F. 156 p.

Riley. J.M.

1988. Gibberellic Acid for Set and Seed Germination. CRFG Journal. 19: 10-12.

Rueda, E.; Castellanos, T.; Troyo, E.; Díaz, A.

2004. Effect of Klebsiella pneumoniae and Azospirillum halopraeferens on the Growth and development of Two Salicornia Bigelovii Genotypes. Australian Journal of Experimental Agriculture, 44 (1) 65-74.

SAS

2015. JMP. Versión 9.1. Institute Inc., Raleigh N.C.

Sokal, R.; Rohfl J.

1988. Biometry: the principles and practice of statistics in biological research. 3nd ed. Freeman \& Co. San Francisco, CA. USA. 887 p.

Ungar, I.

2000. Ecophysiology of vascular halophytes. Department of Botany. Ohio University. Athens Ohio. CRC Press, p. 209. 